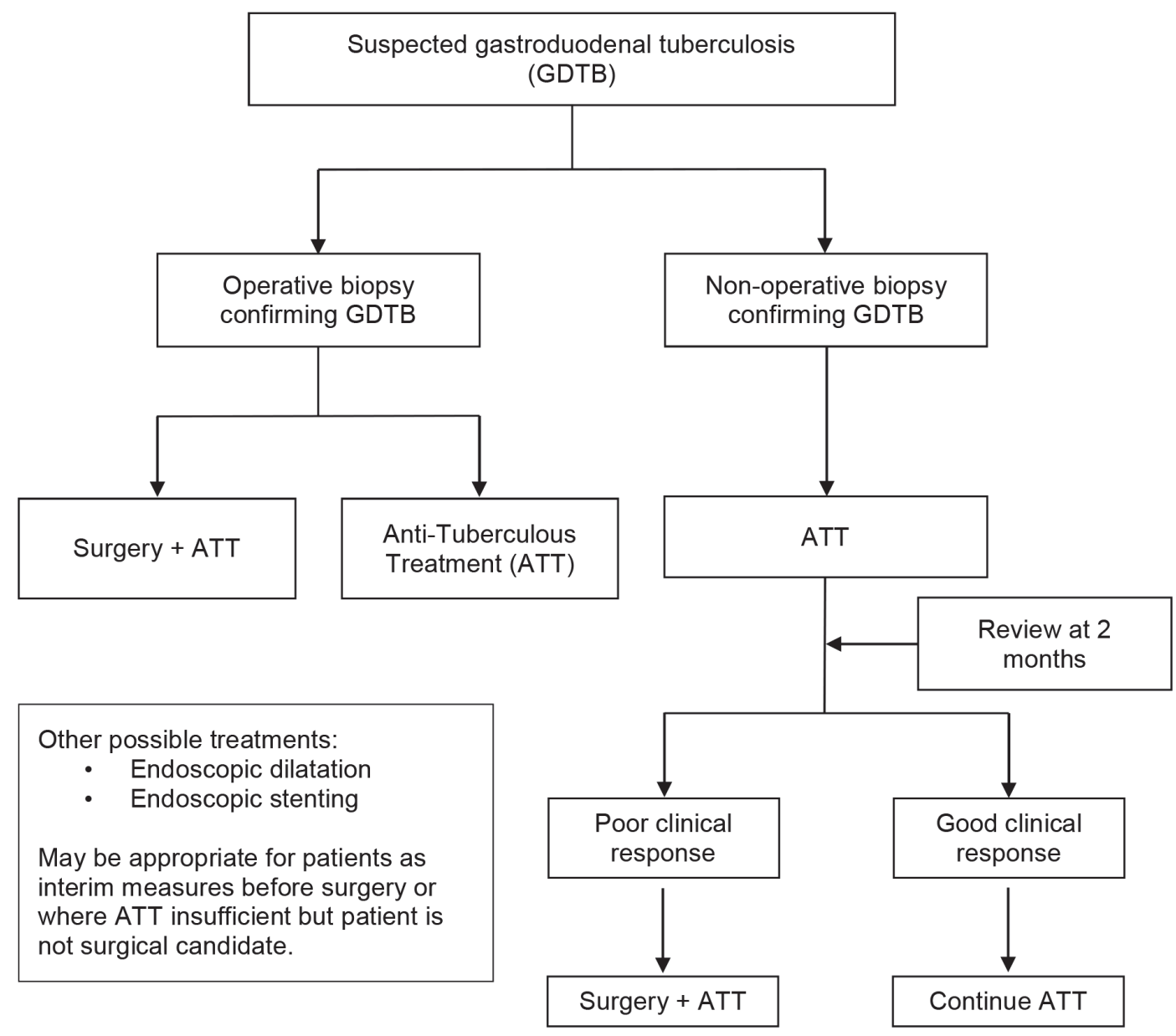

Abstract PTH-85 Figure 1 Management algorithm for clinicians treating patients with GDTB

\section{PTH-86 DYSPLASIA IN SPORADIC FUNDIC GLAND POLYPS: RATE AND MORBIDITY}

Riad Alame*, Ralph Boulton, Michael Ding, Syazeddy Samani, Adil Butt. University Hospital Birmingham, Birmingham, UK

\subsection{6/gutjpl-2021-BSG.265}

Introduction Literature indicates the rate of dysplasia in Fundic Gland Polyps (FGP) is $1 \%$ in non-familial adenomatous polyposis syndrome (FAP) patients. ${ }^{1}{ }^{2}$ The 2019 British Society Guideline (BSG) advise sampling any stomach polyps (SP) and to perform polypectomy for polyps $\geq 1 \mathrm{~cm} .{ }^{3} 4$ We aimed to assess the rate of dysplasia in sporadic FGP and its association with proton pump inhibitors (PPI).

Methods A retrospective \& prospective cohort study of patients with SP diagnosed between 2015 - 2021 in a single tertiary referral centre. We captured detail on patient demographics, referral indication, endoscopy findings, biopsy results, \& PPI usage. FGP's in patients with FAP were excluded.

Results 181 patients with SP of whom 103 (57\%) were sporadic FGP (mean age $70.71 \pm 12.56$; Female to male ratio 1:31). Of these, 5/181 (4.8\%) had dysplasia (4 low grade dysplasia (LGD); 1 high grade dysplasia (HGD)). Only 58\% of sporadic FGPs were biopsied. In the 78 (43\%) non-FGPs; (mean age $70.44 \pm 13.05$; Female to male ratio 1.48), 6 (7.7\%) had LGD and $1(1.3 \%)$ had cancer. Indications for referral were melaena/haematemesis 50(27\%), anaemia 43 (24\%), dysphagia 16(9\%) and dyspepsia 14(8\%). Most of the patients (176/181) were on PPI before the index endoscopy. Lansoprazole was the most common PPI 110(62.5\%), followed by Omeprazole 51(29\%) and Esomeprazole 15(8.5\%). The duration of PPI prescription was between 4-229 months. Patients who developed LGD in FGP were on PPI for at least 9 years. One patient with HGD in FGP was on PPI for 19 years. None of the FGP patients had cancer secondary to polyps, in contrast to one patient with adenocarcinoma in nonFGP.

Conclusion Our study showed the rate of dysplasia to be higher than previously published. The risk of dysplasia development may be associated with prolonged PPI usage $(>8$ years) in our cohort. Our practice is not yet in line with BSG guidelines due to service implications of this recommendation of sampling all polyps. Primary care should review and stop PPI where necessarily given its association with $\mathrm{FGP}^{6}{ }^{7}$

\section{REFERENCES}

1. Levy MD. Sporadic FGP with LGD: A Large Case Series Evaluating Pathologic and Immunohistochemical Findings and Clinical Behavior. Am J Clin Pathol. 2015 Oct; 144(4).

2. Gastric FGP. R W BURT. GASTROENTEROLOGY 2003; 125.

3. Goddard AF. The management of gastric polyps. Gut. 2010 Sep;59(9).

4. Banks M. Diagnosis \& management of patients at risk of gastric adenocarcinoma Gut. 2019 Sep;68(9).

5. Islam RS. Gastric polyps: a review of clinical, endoscopic, and histopathologic features and management decisions. Gastroenterol Hepatol (N Y). 2013 Oct;9(10).

6. Tran-Duy A. Use of PPI and Risks of FGP and Gastric Cancer: Systematic Review and Meta-analysis. Clin Gastro Hepatol. 2016 Dec;14(12).

7. Martin FC. Systematic review with meta-analysis: FGP and PPI. Aliment Pharma Ther. 2016 Nov; 44(9). 